\title{
INTERFACES ENTRE ALIMENTAÇÃO ESCOLAR E AGRICULTURA FAMILIAR: REVISÃO BIBLIOMÉTRICA E DE CONTEÚDO BIBLIOGRÁFICO SOBRE A EXPERIÊNCIA BRASILEIRA
}

\author{
Sandro Pereira Silva ${ }^{1}$
}

\begin{abstract}
RESUMO
Este estudo aborda a produção científica sobre a relação entre Estado, alimentação escolar e agricultura familiar no Brasil, antes (a partir de 1974) e após a reformulação do Programa Nacional de Alimentação Escolar (PNAE) em 2009. Para isso, foi realizado um levantamento bibliométrico no intuito de traçar um panorama quantitativo das pesquisas de interesse, apresentadas quanto a seus períodos de publicação, áreas de conhecimento, canais de divulgação, abordagens metodológicas e palavraschave mais utilizadas. Posteriormente, realizou-se uma categorização temática de todos os trabalhos selecionados, visando identificar os principais assuntos enfatizados, para então destacar as evidências gerais encontradas e sistematizadas a partir de uma revisão qualitativa de seu conteúdo. Assim, obteve-se uma aproximação privilegiada da operacionalização do PNAE, demonstrando aspectos concretos sobre os desafios e os potenciais associados à inserção da agricultura familiar em mercados institucionais, ao mesmo tempo em que enfatizam fatores possíveis para a potencialização desse instrumento no território brasileiro.
\end{abstract}

Palavras-chave: alimentação escolar, agricultura familiar, compras públicas, segurança alimentar e nutricional, PNAE.

\section{INTERFACES BETWEEN SCHOOL FEEDING AND FAMILY AGRICULTURE: BIBLIOMETRIC AND BIBLIOGRAPHIC CONTENT REVIEW ON THE BRAZILIAN EXPERIENCE}

\begin{abstract}
This study addresses scientific production on the relationship between state, school feeding and family agriculture in Brazil, before and after the reformulation of the National School Feeding Program (PNAE) in 2009. For this purpose, a bibliometric survey was conducted in order to draw a quantitative overview of research of interest, presented as to its publication periods, areas of knowledge, dissemination channels, methodological approaches and keywords most used. Subsequently, a thematic categorization of all selected works was performed, aiming at identifying the main emphasized subjects, to then highlight the general evidences found and systematized from a qualitative review of its content. Thus, a privileged approach to the operationalization of the PNAE was obtained, demonstrating concrete aspects on the challenges and potentials associated with the insertion of family agriculture in
\end{abstract}

\footnotetext{
${ }^{1}$ Graduação e Mestrado em Economia (UFV). Doutorado em Políticas Públicas, Estratégia e Desenvolvimento (UFRJ). Pesquisador do Instituto de Pesquisa Econômica Aplicada (IPEA). E-mail: sandro.pereira@ipea.gov.br
} 
institutional markets, while emphasizing possible factors for the potentiation of this instrument in the Brazilian territory.

Keywords: school feeding, family agriculture, public procurement, food and nutritional security, PNAE.

\section{INTRODUÇÃO}

O tema da alimentação escolar no Brasil pode ser considerado um domínio de intervenção governamental estruturado em torno do Programa Nacional de Alimentação Escolar (PNAE). Ele deriva de uma longa trajetória de construção institucional cuja origem remete ao cenário pós $2^{\text {a }}$ Guerra Mundial e, desde então, tem assumido desenhos operacionais variados que se moldam de acordo com as condições políticas e econômicas do Estado brasileiro (Silva, 2019).

Presente em todos os municípios do país, o PNAE é reconhecido como um dos maiores programas de alimentação do mundo, alcançando uma média diária superior a 40 milhões de estudantes em período escolar regular. Tal magnitude lhe confere caráter estratégico para assegurar níveis satisfatórios de segurança alimentar e nutricional em todas as unidades da federação, além de contribuir para o Direito Humano à Alimentação Adequada (DHAA) da população (D'AVILA; SILVA, 2011; IPEA, 2020; SILVA, 2014b; SILVA; VALADARES, 2018).

Embora a gestão política do programa seja concentrada na União, responsável pela normatização geral e monitoramento de sua implementação, a execução financeira ocorre de forma descentralizada. O Fundo Nacional de Desenvolvimento da Educação (FNDE), autarquia federal responsável por sua gestão, repassa recursos orçamentários aos demais entes federativos para a compra de gêneros alimentícios, cujos valores são calculados em função do número de estudantes matriculados na rede pública de ensino. ${ }^{2}$ Nesse processo, destaca-se o papel dos Conselhos de Alimentação Escolar (CAE), que atua no acompanhamento e controle social a nível municipal dos gastos e do atendimento do conjunto de normas estabelecidas pelo FNDE.

Em 2009, com a Lei no 11.947, o PNAE passou por uma significativa reformulação. Entre as novas diretrizes instituídas está a obrigatoriedade de os entes federativos destinarem no mínimo $30 \%$ dos recursos repassados pelo FNDE para a aquisição de gêneros alimentícios diretamente da agricultura familiar (art. 14).

O segmento socioprodutivo da agricultura familiar representa um amplo contingente de trabalhadores brasileiros que guardam entre si significativas especificidades associadas ao contexto territorial no qual se inserem (Silva, 2015; 2016; Pacífico, 2017). A Lei no 11.326/2006 caracteriza esse público como aquele que pratica atividades no meio rural e atende aos seguintes requisitos: $l$ ) não detenha área maior que quatro módulos fiscais; ii) utilize predominantemente força de trabalho da própria família; iii) tenha renda familiar predominantemente originada de atividades econômicas vinculadas ao próprio estabelecimento; e iv) dirija o estabelecimento rural em regime de economia familiar.

A nova regra de compra prevista pela Lei no 11.947/2009 abriu um canal de acesso bastante atrativo ao mercado institucional de alimentos a esse público por meio da dispensa de processos licitatórios padrão, regulamentando via resoluções posteriores um novo procedimento de compras, denominado "chamada pública",

\footnotetext{
2 Os valores de referência são definidos pela Resolução CD/FNDE n. 1/2017, e vão de $R \$ 0,32$ (educação de jovens e adultos - EJA) a R\$1,07 (educação em tempo integral) (SIL̄VA, 2019).
} 
exclusiva para a aquisição de gêneros de alimentos destinados à alimentação escolar. ${ }^{3}$ Com isso, o programa passou a compor um rol de políticas públicas federais voltadas à agricultura familiar em todo o território nacional, a exemplo do Programa Nacional de Fortalecimento da Agricultura Familiar (Pronaf) e o Programa de Aquisição de Alimentos (PAA) (GRISA; SCHNEIDER, 2014; SAMBUICHI, 2019; SILVA, 2014a; VALADARES et al., 2020).

No entanto, as unidades federativas precisaram se adaptar para absorver em seus processos administrativos as novas sistemáticas de compra. Esse aprendizado é refletido na evolução da participação da agricultura familiar nesse mercado. Embora os dados oficiais indiquem que a média nacional ainda não atingiu o mínimo previsto de $30 \%$ dos repasses do FNDE, é possível perceber um aumento gradativo ao longo dos anos. ${ }^{4}$ Enquanto em 2010, primeiro ano de vigência da lei, essa média foi de apenas 4,9\%, desde 2015 ela supera o patamar dos $20 \%$, o que representa algo próximo a $\mathrm{R} \$ 1$ bilhão negociados diretamente com agricultores familiares (SILVA, 2019).

Toda essa interface entre alimentação escolar e agricultura familiar propiciada pela Lei no 11.947/2009 pode ser problematizada sob uma miríade de dimensões, dado o caráter intersetorial que caracteriza tanto o PNAE (para além do setor educacional, envolvendo saúde, assistência social, entre outras) quanto o próprio público dos agricultores familiares (considerando sua relevância para a discussão de temas como segurança alimentar, meio ambiente, geração de renda, etc.). ${ }^{5}$ Torna-se então razoável supor, como hipótese, que essa maior complexidade institucional tenha despertado o interesse de novos pesquisadores em distintos campos de conhecimento científico, de modo que uma parcela maior das pesquisas que tem como foco empírico o PNAE passe a explorar essa nova dimensão de análise.

Nessa perspectiva, este trabalho visou analisar a produção científica sobre a relação entre Estado, alimentação escolar e agricultura familiar no Brasil, antes e após a Lei $\mathrm{n}$-11.947/2009. O objetivo foi verificar como esses termos têm sido abordados na literatura, averiguando a diversidade de problematizações possíveis que a interface entre eles suscita.

Para isso, na seção 2 foi realizado um levantamento bibliométrico no intuito de traçar um panorama quantitativo da produção de interesse. Na seção 3, optou-se por realizar uma categorização temática de todos os trabalhos selecionados, visando identificar os principais assuntos enfatizados, para então destacar as evidências gerais encontradas e sistematizadas a partir de uma revisão qualitativa de seu conteúdo bibliográfico. Na seção 4 apresenta-se uma síntese das evidências encontradas na literatura consultada. Por fim, são tecidas algumas considerações conclusivas.

\section{PERCURSO METODOLÓGICO E PANORAMA GERAL DE ANÁLISE}

A técnica de pesquisa bibliométrica tem como função contribuir para uma avaliação quantitativa de um fluxo de informações bibliográficas para traçar o "estado da arte" da produção científica sobre um determinado tema, destacando padrões e tendências utilizados pelos autores para a geração de indicadores que auxiliem no tratamento analítico. Seu uso tem sido cada vez mais recorrente em todas as grandes

\footnotetext{
${ }^{3}$ Resolução/CD/FNDE no. 38/2009 e Resolução/CD/FNDE/MEC nํ. 4/2015.

4 A Lei n. 11.947/2009 também estabeleceu flexibilizações para o caso de entes federativos que não alcançarem o patamar indicado de compras da agricultura familiar (SILVA, 2019).

${ }^{5}$ Para uma discussão sobre a multifuncionalidade da agricultura familiar, ver Silva (2016).
} 
áreas do conhecimento, inclusive em avaliações sobre questões de intervenção estatal (SILVA, 2018; VIEIRA, 2019).

Para este trabalho, realizou-se um levantamento em periódicos indexados de três portais de acesso livre: o Scientific Periodicals Eletronic Library (Spell), o Scientific Electronic Library Online (Scielo) e o repositório do Instituto de Pesquisa Econômica Aplicada (Ipea). Em termos de abrangência temporal, as publicações se situam entre 1974, ano do primeiro artigo identificado, até 2019. A seleção inicial de artigos se deu com base nos seguintes descritores: alimentação escolar "ou" PNAE, contidos no assunto (título, palavras-chave ou resumo), em periódicos revisados por pares, independentemente da área de conhecimento.

Nessa rodada foram identificados 275 artigos completos. Daí então foi realizado um segundo filtro de busca, verificando nos campos título, resumo e palavras-chave quais desses artigos abordam de alguma forma a temática da produção agrícola familiar (agricultura familiar, produção local, regional, pequena produção agrícola e outros temas associados) em conexão com a questão da alimentação escolar. Ao fim, foram selecionados 79 artigos para a análise bibliométrica pretendida.

Uma primeira observação relevante, ancorada na distribuição temporal dessas publicações, é que a Lei no 11.947/2009 impactou na diversificação e expansão do interesse dos pesquisadores sobre o programa. Basta ver a participação relativa dos artigos sobre o PNAE que abordam a questão da produção agrícola familiar para o fornecimento de gêneros alimentícios ao longo do tempo, considerando o universo inicial de artigos identificados $(n=275)$. Enquanto até o ano de 2009 apenas $10,7 \%$ das publicações encontradas apresentavam alguma associação analítica com a agricultura familiar, entre os anos de 2010 e 2019 esse percentual subiu para $33,3 \%$. No geral, considerando todo o horizonte temporal da pesquisa, $28,7 \%$ dos artigos identificados sobre a temática da alimentação escolar estão associados ao tema da agricultura familiar e compras localizadas de alimentos (tabela 1).

Outro ponto a destacar é quanto à diversificação de áreas de conhecimento cobertas pelos respectivos periódicos, entre as quais se encontram: economia, sociologia, administração, geografia, gestão pública, extensão rural, além de periódicos de caráter multidisciplinar. Tomando como base os 79 artigos filtrados para a análise bibliométrica, foi possível identificar 23 periódicos diferentes, sendo que os dois principais seguiram sendo nas áreas de ciências da nutrição e saúde, conforme demonstra a tabela 2, que lista os periódicos com mais de um artigo identificado. Todos os textos identificados são listados nas referências finais. 
Tabela 1 - Distribuição temporal dos artigos identificados para a pesquisa bibliométrica

\begin{tabular}{cccc}
\hline Períodos & $\begin{array}{c}\text { (a) Total inicial de } \\
\text { artigos } \\
\text { identificados }\end{array}$ & $\begin{array}{c}\text { (b) Artigos com a } \\
\text { temática da agricultura } \\
\text { familiar }\end{array}$ & $\begin{array}{c}\text { \% de artigos sobre } \\
\text { agricultura familiar (a / b) }\end{array}$ \\
\hline $1974-1999$ & 24 & 1 & 4,2 \\
$2000-2009$ & 32 & 5 & 15,6 \\
2010 & 11 & 1 & 9,1 \\
2011 & 14 & 2 & 14,3 \\
2012 & 17 & 3 & 17,6 \\
2013 & 35 & 10 & 28,6 \\
2014 & 22 & 9 & 40,9 \\
2015 & 20 & 8 & 40,0 \\
2016 & 19 & 5 & 26,3 \\
2017 & 24 & 9 & 37,5 \\
2018 & 24 & 7 & 29,2 \\
2019 & 33 & 19 & 56,3 \\
\hline Até 2009 & 56 & 6 & 10,7 \\
Pós 2009 & 219 & 73 & 33,3 \\
\hline Total & 275 & 79 & 28,7 \\
\hline
\end{tabular}

Fonte: Elaboração do autor.

Tabela 2 - Principais periódicos identificados por número de artigos selecionados

\begin{tabular}{ccc}
\hline Periódico & Total de artigos selecionados & $\%$ \\
\hline Revista de Nutricão & 17 & 21,5 \\
Revista Ciência e Saúde Coletiva & 13 & 16,5 \\
Revista de Economia e Sociologia Rural & 9 & 11,4 \\
Revista Redes & 9 & 11,4 \\
Revista Ciência Rural & 4 & 5,1 \\
Cadernos de Saúde Pública & 3 & 3,8 \\
Revista Desenvolvimento em Questão & 3 & 3,8 \\
Revista Espacios & 3 & 3,8 \\
Revista Planejamento e Políticas Públicas & 2 & 2,5 \\
Revista Interface & 2 & 2,5 \\
Revista Saúde e Sociedade & 2 & 2,5 \\
\hline
\end{tabular}

Fonte: Elaboração do autor.

Para a análise conjunta dos artigos selecionados, buscou-se caracterizá-los inicialmente de acordo com as metodologias adotadas por seus autores, dividindo as respectivas pesquisas entre aquelas com abordagem predominantemente qualitativa e as demais de abordagem quantitativa. Conforme observado, a maioria delas partiu de análises de natureza qualitativa, 50 (63,3\% do total), enquanto 29 (36,7\%) optaram pela abordagem quantitativa. O gráfico 1 demonstra a evolução dessas publicações ao longo do tempo, com destaque para o crescimento das publicações em 2019. 
Gráfico 1 - Evolução da produção bibliográfica por tipo de abordagem metodológica

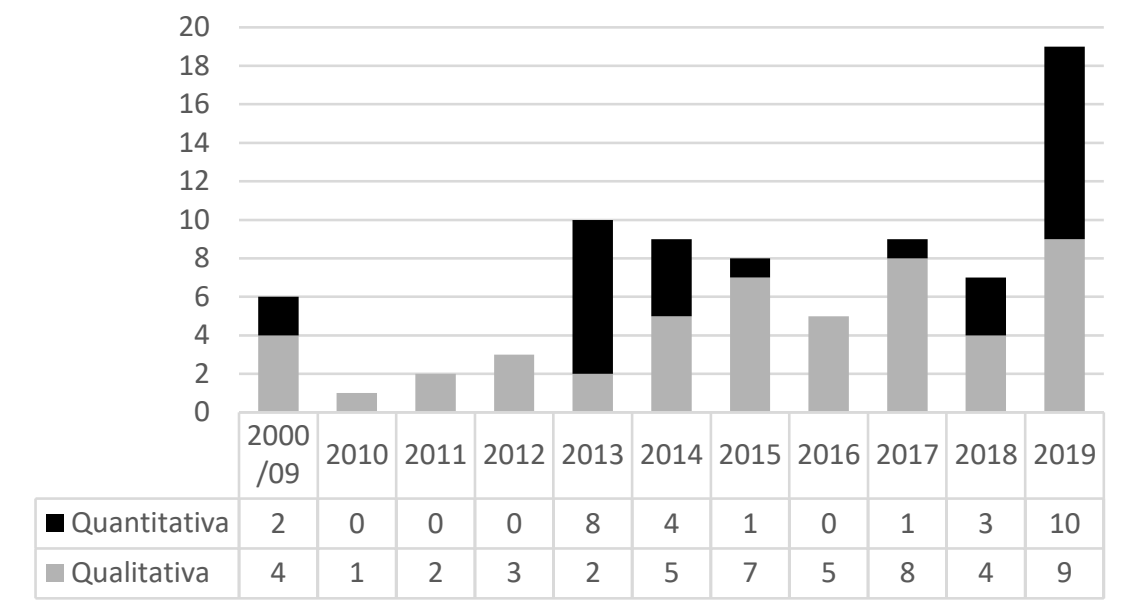

Fonte: Banco de dados da pesquisa. Elaboração do autor.

Entre os artigos com abordagem qualitativa, predominam aqueles baseados em estudos de caso - de caráter exploratório, descritivo ou comparativos. Esses estudos visam, de maneira geral, analisar aspectos operacionais diversificados da Lei no 11.947/2009 a partir de realidades territoriais (sobretudo em domínios municipais) específicas, seja para captar e descrever a dinâmica processual do PNAE, seja para enfatizar elementos particulares e inovadores. Quanto aos artigos com abordagem quantitativa, a maioria envolveu inferências estatísticas a partir de tabulações de dados primários e secundários, baseados em dados orçamentários do PNAE, registros administrativos e levantamentos específicos junto a informantes-chave.

Outra caracterização do material selecionado refere-se ao conteúdo e à frequência das palavras-chave de cada texto. Foram identificadas 92 palavras-chave diferentes, sendo que as duas que apareceram mais vezes foram justamente 'alimentação escolar', com 47 indicações, e 'agricultura familiar', com 39, ou seja, $59,5 \%$ e $49,4 \%$ dos textos selecionados, respectivamente. A tabela 3 lista as palavraschave com pelo menos cinco aparições.

Tabela 3 - Principais palavras-chave identificadas por número de artigos selecionados

\begin{tabular}{ccc}
\hline Palavras-chave & Total & $\%$ \\
\hline Alimentação escolar & 46 & 59,5 \\
Agricultura familiar & 39 & 49,4 \\
Políticas Públicas & 25 & 32,9 \\
Segurança alimentar e nutricional & 18 & 24,1 \\
Programas e políticas de nutrição e alimentação & 12 & 15,2 \\
Desenvolvimento rural & 8 & 10,1 \\
Alimentos orgânicos & 5 & 7,6 \\
Desenvolvimento local & 5 & 6,3 \\
Mercados institucionais & 5 & 6,3 \\
Abastecimento de alimentos & 5 & 6,3 \\
Cooperativismo & 5 & 6,3 \\
\hline
\end{tabular}

Fonte: Banco de dados da pesquisa. Elaboração do autor. 


\section{CATEGORIAS TEMÁTICAS E CONTEÚDO ANALÍTICO}

No intuito de se obter uma visão mais detalhada da multiplicidade de fatores debatidos, procurou-se classificar os principais objetos de análise nos artigos em um exercício de categorização. Para isso, foi definido um assunto central como categoria temática para cada um deles. Para os casos mais difíceis de definir um assunto específico, o esforço foi no sentido de classificá-los dentro do tema que mais se enquadrava, de acordo com os objetivos desta pesquisa. A leitura dos textos permitiu a identificação e seu agrupamento em doze categorias temáticas, conforme demonstrado na tabela 4 , com suas respectivas referências.

Tabela 4 - Assuntos principais de pesquisa e suas referências

\begin{tabular}{|c|c|c|}
\hline Categorias & Referências & Total \\
\hline Compras públicas & $\begin{array}{l}\text { Andrade, Araújo e Santos (2019); Araújo et al. (2019); Baccarin } \\
\text { et al. (2017); Bezerra et al. (2013); Corrêa et al. (2019); Ferigollo } \\
\text { et al. (2017); Machado et al. (2018); Ribeiro, Seratti e Broch } \\
\text { (2013); Sá et al. (2017); Saraiva et al. (2013); Triches e Kilian } \\
\text { (2016); Triches e Silvestri (2018); Villar et al. (2013); Vilela et al. } \\
\text { (2019) }\end{array}$ & 14 \\
\hline $\begin{array}{l}\text { Arranjos } \\
\text { organizacionais e } \\
\text { desenvolvimento } \\
\text { local }\end{array}$ & $\begin{array}{l}\text { Cunha, Freitas e Salgado (2017); Elias et al. (2019); Franzoni e } \\
\text { Silva (2016); Freitas, Ferreira e Freitas (2019); Martins e Lima } \\
\text { (2017); Oliveira, Batalha e Pettan (2017); Silva (2014a); Souza- } \\
\text { Esquerdo e Bergamasco (2014); Triches e Schneider (2010); } \\
\text { Turpin (2009); Wagner, Gehlen e Shult (2016) }\end{array}$ & 11 \\
\hline $\begin{array}{l}\text { Condições de } \\
\text { abastecimento e } \\
\text { comercialização }\end{array}$ & $\begin{array}{l}\text { Amaral et al. (2018); Antunes e Vivaldini (2018); Fernandes e } \\
\text { Engel (2014); Marques et al. (2014); Monego et al. (2013); } \\
\text { Mossmann et al. (2017); Schabarum e Triches (2019); Soares et } \\
\text { al. (2015); Triches et al. (2019); Vianna e Tereso (2000); Vilhena, } \\
\text { Alves e Freitas (2019) }\end{array}$ & 11 \\
\hline $\begin{array}{l}\text { Gestão pública } \\
\text { educacional }\end{array}$ & $\begin{array}{l}\text { Bandeira et al. (2013); Basso, Lopes e Amaral (2019); Belik e } \\
\text { Chaim (2009); Carvalho (2009); Gabriel, Goulart e Calvo (2015); } \\
\text { Kroth et al. (2019); Lopes e Doula (2016; 2019); Melo, Sá e Melo } \\
\text { Filho (2016); Real e Schneider (2011); Souza e Villar (2019) }\end{array}$ & 11 \\
\hline $\begin{array}{l}\text { Alimentação e } \\
\text { saúde pública }\end{array}$ & $\begin{array}{l}\text { Chaves et al. (2009); Gabriel et al. (2012); Gonçalves et al. } \\
\text { (2015); Paiva, Freitas e Santos (2012); Siqueira et al. (2014); } \\
\text { Soares et al. (2018); Teo e Monteiro (2012); Trivellato et al. } \\
\text { (2019) }\end{array}$ & 8 \\
\hline $\begin{array}{l}\text { Produção } \\
\text { agroecológica e } \\
\text { sustentabilidade }\end{array}$ & $\begin{array}{l}\text { Lima e Sousa (2011); Santos et al. (2014); Maluf et al. (2015); } \\
\text { Nascimento et al. (2019); Silva e Sousa (2013); Silverio e Sousa } \\
\text { (2014); Sousa et al. (2015) }\end{array}$ & 7 \\
\hline $\begin{array}{l}\text { Cooperativismo e } \\
\text { associativismo }\end{array}$ & $\begin{array}{l}\text { Araújo et al. (2017); Costa, Amorim e Silva (2015); Estevam, } \\
\text { Salvaro e Santos (2018); Silva, Dias e Amorim (2015) }\end{array}$ & 4 \\
\hline Estrutura escolar & $\begin{array}{l}\text { Fernandes, Fonseca e Silva (2014); Ferreira et al. (2019); } \\
\text { González-Chica et al. (2013) }\end{array}$ & 3 \\
\hline Marco legal & Peixinho (2013); Schwartzman et al. (2017); Silva (2019) & 3 \\
\hline $\begin{array}{l}\text { Comparação } \\
\text { internacional }\end{array}$ & Belik e Souza (2009); Grisa e Calderón (2019); Swensson (2019) & 3 \\
\hline $\begin{array}{l}\text { Comunidades } \\
\text { tradicionais }\end{array}$ & Carvalho e Silva (2014); Sousa et al. (2013) & 2 \\
\hline $\begin{array}{l}\text { Revisão de } \\
\text { literatura }\end{array}$ & Libermann e Bertolini (2015); Pedraza et al. (2018) & 2 \\
\hline
\end{tabular}

Fonte: Banco de dados da pesquisa. Elaboração do autor. 
Segue então um mapeamento sintético dos conteúdos e evidências trazidas pelas produções enquadradas em cada uma das categorias elencadas.

\subsection{COMPRAS PÚBLICAS}

A principal categoria identificada entre as pesquisas que envolvem a relação entre PNAE e agricultura familiar enfatiza justamente a questão das compras públicas, isto é, a verificação da situação das entidades executoras e a problematização de particularidades quanto ao atendimento da regra de compra de produtos da agricultura familiar prevista pela Lei $n=11.947 / 2009$. Todos os quatorze textos dessa categoria foram publicados a partir de 2013 e envolveram pesquisas com diferentes escalas territoriais de análise - nacional (pesquisas transversais sobre o conjunto de municípios brasileiros), estadual e grupos de municípios.

As principais abordagens identificadas foram: i) panoramas nacionais quanto ao cumprimento da regra de compra da agricultura familiar (Saraiva et al. 2013; Machado et al. 2018; Araújo et al., 2019); ii) desempenho municipal em estados específicos (BACCARIN et al., 2017; VILLAR et al., 2013; TRICHES; SILVESTRI; 2018; TRICHES; KILIAN, 2016; FERIGOLLO et al., 2017; CORRÊA et al., 2019; RIBEIRO; CERATTI; BROCH, 2013; ANDRADE; ARAÚJO; SANTOS, 2019; SÁ et al., 2017; BEZERRA et al., 2013); e iii) execução orçamentária do PNAE nos campi do Instituto Federal de Educação, Ciência e Tecnologia Baiano (Vilela et al., 2019).

De acordo com os resultados observados, embora o percentual médio de compra alcançado no conjunto dos municípios ainda esteja abaixo do estabelecido, há uma evolução considerável para a progressiva efetivação desta normativa nos diferentes contextos brasileiros, tanto em termos de número de fornecedores quanto de escolas atendidas. A elevação da participação da agricultura familiar tem garantido a diversidade de alimentos adquiridos, especialmente dos grupos 'hortaliças, legumes e verduras', 'cereais, pães, massas e tubérculos' e 'frutas', sendo a maior parte produtos in natura. Contudo, os indicadores se expressam de maneira bastante heterogênea no país, dada a existência de múltiplas particularidades locais em termos de fatores facilitadores e limitantes, o que dificulta o estabelecimento de padrões.

Entre as dificuldades encontradas nos municípios investigados originavamse tanto do lado da oferta (dificuldades dos agricultores para o fornecimento regular e adequação dos produtos) quanto da demanda, explicado por particularidades na dinâmica da gestão pública em cada um dos municípios (falta de preparo e desconhecimento por parte dos gestores de seu papel no processo, falta de empenho das prefeituras na elaboração e divulgação das chamadas públicas). Tais fatores prejudicam a eficiência do instrumento para a aquisição dos produtos aos quais ele se destina, dificultando, assim, que o PNAE alcance seus objetivos de promover alimentação adequada e desenvolvimento sustentável. Outras variáveis com influência na decisão de adquirir esses produtos foram: população do município, tipo de gestão da alimentação escolar, presença de nutricionista como responsável técnico e grau de organização coletiva dos agricultores.

Em termos de recomendações, os autores propõem fortalecer a articulação de redes intersetoriais capazes de desencadear processos locais visando à promoção de produtos da agricultura familiar, sobretudo no tocante à organização coletiva dos agricultores para o atendimento desse mercado, na sensibilização de gestores municipais e na contratação de nutricionistas por parte das prefeituras. 


\subsection{ARRANJOS ORGANIZACIONAIS E DESENVOLVIMENTO LOCAL}

A segunda categoria temática foi a de arranjos organizacionais e desenvolvimento local, com onze artigos. Nessa categoria estão agregados os textos que abordam diferentes articulações em nível local/territorial de desenvolvimento desencadeados a partir da viabilização do mercado institucional do PNAE para a agricultura familiar.

Foi possível observar que mesmo antes da Lei 11.947/2019 já havia experiências de integração da agricultura familiar e o mercado institucional da alimentação escolar (TURPIN; 2009; TRICHES; SCHNEIDER, 2010). Contudo, as regulamentações eram muito contraditórias, como no caso de processos licitatórios e exigências legais e sanitárias que inviabilizavam a participação desses agricultores.

Nesse sentido, a nova normatização do PNAE, ao estabelecer reserva importante de mercado para esse segmento socioprodutivo com acesso restrito até então à circulação de valores via compras públicas, abriu um espaço importante para sua incorporação no fornecimento de produtos para a alimentação escolar, além de favorecer a viabilização de cadeias agroalimentares curtas (Franzoni e Silva, 2016). Essa incorporação ocorreu, como já citado anteriormente, de forma heterogênea no plano nacional, o que implica dinâmicas de articulação social e arranjos organizacionais diferenciadas de acordo com contextos territoriais específicos que afetam diretamente os resultados do programa (SILVA, 2014a; FREITAS, FERREIRA; FREITAS, 2019; WAGNER; GEHLEN; SCHULT, 2016).

Alguns estudos analisaram também os efeitos conjuntos de políticas de compras públicas da agricultura familiar (PNAE, PAA e programas estaduais), uma vez que há grande sobreposição entre os agentes envolvidos (SOUZA-ESQUERDO; BERGAMASCO, 2014; OLIVEIRA; BATALHA; PETTAN, 2017; MARTINS; LIMA, 2017; CUNHA; FREITAS; SALGADO, 2017; ELIAS et al., 2019). De modo geral, os estudos identificaram os seguintes efeitos potencializadores desses programas na dinâmica socioeconômica da agricultura familiar (processos produtivos e produtos): $i$ ) econômico: aumento de produção, trabalho e renda; diversificação produtiva e acesso a novos mercados; ii) social: fortalecimento das organizações associativas locais, ampliação das relações institucionais, maior envolvimento familiar na produção; iii) meio ambiente: o incentivo à produção livre de agrotóxico; e iv) segurança alimentar: melhoria na alimentação das famílias e aumento da qualidade dos produtos.

Os autores reforçam a necessidade constante de monitoramento e ajustes na operacionalidade do programa para contemplar a heterogeneidade e a dispersão espacial da agricultura familiar nos distintos territórios em que se inserem.

\subsection{CONDIÇÕES DE ABASTECIMENTO}

A terceira categoria foi sobre condições de abastecimento e comercialização. Os onze textos identificados versam sobre aspectos que limitam ou potencializam a relação da agricultura familiar com a execução do PNAE, sobretudo em função da capacidade de produção e distribuição de alimentos.

Esses estudos partiram tanto da análise estrutural para o atendimento das demandas institucionais (estruturas de armazenamento e distribuição de alimentos, capacidade de agroindustrialização) (Vianna e Tereso, 2000; Soares et al., 2015; Fernandes e Engel, 2014; Amaral et al., 2018), quanto de percepções dos atores sociais envolvidos (agricultores familiares, gestores escolares, profissionais de extensão rural, membros de CAEs, nutricionistas, entre outros) para problematizar a dinâmica operacional do PNAE e sua relação com o universo de práticas da agricultura familiar (MARQUES et al., 2014; MOSSMANN et al., 2017; ANTUNES; VIVALDINI, 
2018; TRICHES et al., 2019; SCHABARUM; TRICHES, 2019). Houve ainda estudos que problematizaram sobre a participação dos produtos da sociobiodiversidade na pauta de programas de compras públicas de agricultores familiares, considerando seu potencial de indução ao desenvolvimento regional sustentável (MONEGO et al., 2013; VILHENA; ALVES Jr; FREITAS, 2019).

As análises perpassaram distintas questões identificadas nas pesquisas empíricas, tais como: dificuldades referentes ao pagamento dos produtos entregues, ao transporte e à estrutura física disponibilizada, assistência técnica insuficiente, fragilidades na organização coletiva dos agricultores e falta de conhecimento e/ou interesse por parte dos gestores públicos, entre outros fatores que se expressam de forma diferenciada nos municípios, o que demonstra a complexidade na implementação do programa em diferentes contextos territoriais. Na opinião dos autores, o enfrentamento desses obstáculos deve passar por mecanismos de diálogo, intersetorialidade, investimento, organização coletiva, atividades de capacitação e planejamento integrado de ações visando o atendimento das exigências normativas.

Já os benefícios mais enfatizados referem-se às oportunidades proporcionadas por esse mercado após a Lei no 11.947/2009 e seu potencial de contribuir para o aumento da renda e da qualidade de vida das famílias rurais. Foi reforçada também a necessidade de um equilíbrio na definição dos preços pagos aos agricultores familiares, que devem ser atrativos e justos sem prejudicar a racionalização do gasto público, o que consiste no grande desafio para o sucesso do programa, considerando inclusive suas potenciais externalidades positivas ao território.

\subsection{GESTÃO PÚBLICA EDUCACIONAL}

A quarta categoria a ser destacada é gestão pública educacional, com onze artigos que tratam tanto sobre aspectos mais normativos e burocráticos da gestão da política educacional nos diferentes níveis federativos, dentro da qual se insere 0 PNAE.

Três desses artigos se referem a processos ocorridos anteriormente à reforma do PNAE, em 2009, que destacou a incorporação de processos de "licitações sustentáveis", entendidos como uma prática legal e plausível para auxiliar as localidades e seus respectivos poderes públicos a enfrentarem problemas sociais e economicamente relevantes por meio da prerrogativa das compras públicas, visando atender à demanda escolar (CARVALHO, 2009; BELIK; CHALIM, 2009; REAL; SCHNEIDER, 2011). Nessa perspectiva, o PNAE era vislumbrado como estratégia potencial de desenvolvimento regional sustentável, dado o volume de recursos mobilizados anualmente na aquisição de produtos por estados e municípios. Portanto, ambos os estudos apontavam que o uso das compras públicas na gestão da política educacional em geral, e da alimentação escolar em particular, como elemento de promoção do desenvolvimento local sustentável, já era debatido e até praticado antes de 2009, mesmo que dependente do protagonismo de gestores locais.

Os demais artigos avaliaram os instrumentos de gestão e governança para o atendimento da compra de produtos da agricultura familiar conforme previsto na nova legislação (BANDEIRA et al., 2013; GABRIEL; GOULART; CALVO, 2015; MELO; SÁ; MELO FILHO; 2016; LOPES; DOULA, 2016; 2019; KROTH et al., 2019; BASSO; LOPES; AMARAL, 2019; SOUZA; VILLAR; 2019). Os resultados encontrados por esse conjunto de autores demonstraram que o PNAE tem alcançado seus objetivos em termos de fortalecimento da agricultura familiar em algumas regiões do país, proporcionando melhor qualidade nutricional para os alunos e promovendo o desenvolvimento local. Contudo, foi destacada a necessidade de auxílio logístico e 
assessoria técnica aos agricultores por parte de prefeituras e governos estaduais como forma de potencializar os resultados, o que exige uma gestão intesetorial, apontando o longo caminho pela frente para o atendimento da normativa prevista pela Lei $\mathrm{n}^{\circ}$ 11.947/2009. Ressalta-se também que, em geral, eles identificaram que o tipo de gestão dos recursos (centralizada, descentralizada ou mista) e a frequência de divulgação do processo de compras possuem relevância explicativa no grau de comprometimento com a compra da agricultura familiar.

\subsection{ALIMENTAÇÃO E SAÚDE PÚBLICA}

A quinta categoria foi alimentação e saúde pública, composta por oito artigos que abordam a relação entre PNAE e agricultura familiar sob a perspectiva da segurança alimentar e nutricional dos estudantes que recebem as refeições disponibilizadas, considerando a escola também como um espaço para a valorização da saúde.

Entre os temas identificados estão a influência do PNAE na formação de hábitos alimentares (TEO; MONTEIRO, 2012; PAIVA; FREITAS; SANTOS, 2012; SIQUEIRA et al., 2014; SOARES et al.; 2018), o planejamento dos cardápios de alimentação escolar da rede municipal (CHAVES et al., 2009; GABRIEL et al., 2012; GONÇALVES et al., 2015) e a situação de segurança alimentar em famílias de agricultores fornecedores de produtos para o PNAE (TRIVELLATO et al., 2019).

Os estudos demonstram a existência de diferenças significativas nas sistemáticas locais de definição de cardápios para o PNAE: desde cardápios padronizados e com baixa oferta de frutas/hortaliças até cardápios elaborados segundo critérios da gestão e particularidades das escolas, com maior oferta de frutas/hortaliças. Em geral, os autores apontaram para a necessidade de impulsionar a inclusão de alimentos produzidos regionalmente nos cardápios, viabilizando a entrada dos pequenos produtores locais no mercado, como uma estratégia de diversificação dos cardápios e ampliação do valor nutricional da alimentação escolar. Eles reforçam também a importância de os agentes operadores do programa contemplarem e incentivarem as preparações típicas ao hábito alimentar de cada região no cardápio da alimentação escolar.

Assim, é possível apontar para um consenso nesse conjunto de estudos de que a parceria entre a agricultura familiar e a alimentação escolar pode ser um caminho importante para a promoção de hábitos alimentares saudáveis, não só pelo oferecimento de cardápios de melhor qualidade nutricional, mas pela execução de atividades de educação alimentar, também previstas na Lei no 11.947/2009. Contudo, eles ressaltam que, embora o marco regulatório seja condição essencial, ele por si não é suficiente para a realização do DHAA, sendo necessárias ações complementares para sua concreta efetivação. Há também a necessidade de monitoramento dos fatores que comprometem a segurança alimentar e nutricional das próprias famílias de agricultores, que são fundamentais na garantia de produtos saudáveis para a alimentação escolar em seus respectivos municípios.

\subsection{PRODUÇÃO AGROECOLÓGICA E SUSTENTABILIDADE}

A sexta categoria com a maior frequência de artigos foi a de produção agroecológica e sustentabilidade, com sete no total. Esses estudos refletem sobre processos de produção de alimentos orgânicos/agroecológicos e seu potencial de inserção em programas de alimentação escolar para promover desenvolvimento regional sustentável e segurança alimentar (LIMA; SOUSA, 2011; SILVA; SOUSA, 2013; SANTOS et al., 2014; SOUSA et al., 2015; NASCIMENTO et al., 2019). Houve 
também a análise de fatores operacionais para facilitar a utilização de alimentos orgânicos da agricultura familiar na alimentação escolar (SILVERIO; SOUSA, 2014) e dos elos entre agricultura e nutrição tendo em conta as estruturas institucionais e o desenho dos programas de aquisição de alimentos no Brasil (MALUF et al., 2015).

As análises identificadas apontam como fatores limitantes a inexistência de um planejamento específico para a compra e uso de alimentos orgânicos nos cardápios, além da dificuldade de alguns fornecedores em atender às necessidades das escolas. Os entraves variam de acordo com o grupo envolvido: no caso dos agricultores familiares, destacam-se as dificuldades para a certificação de seus produtos, além de problemas enfrentados na produção, evidenciando a necessidade de apoio técnico; quanto aos nutricionistas, os relatos foram sobre a baixa oferta de alimentos orgânicos em relação às necessidades de composição dos cardápios escolares; para os gestores das cooperativas, as limitações maiores são referentes ao transporte e à sazonalidade da produção.

Nesse sentido, os autores destacam a necessidade de promover hábitos de consumo e produção de alimentos compatíveis com as novas diretrizes do PNAE, bem como o planejamento de cardápios que contemplem a diversidade e sazonalidade regionais. No campo da gestão pública, é ressaltada a necessidade de padronização e simplificação de exigências burocráticas para o atendimento das demandas locais por esses produtores.

\subsection{COOPERATIVISMO E ASSOCIATIVISMO}

O tema do cooperativismo e associativismo na agricultura familiar perpassou diversos estudos desta revisão. Porém, optou-se por uma categoria específica para destacar a institucionalidade cooperativa/associativa na organização de agricultores para o acesso ao PNAE. Os artigos abordaram questões como: oportunidades de acesso a mercados para cooperativas de agricultura familiar via programas de compras públicas (COSTA; AMORIM; SILVA, 2015; ESTEVAM; SALVARO; SANTOS, 2018); mudanças organizacionais em cooperativas da agricultura familiar para o atendimento das novas regras do PNAE (SILVA; DIAS; AMORIM, 2015; ARAÚJO et al., 2017).

De maneira geral, os estudos identificaram que os processos de organização produtiva e gerencial dos cooperados foram afetados pelo conjunto de regras e procedimentos estabelecidos. Foram identificados alguns fatores que implicam diretamente na capacidade dessas organizações atenderem às exigências do programa, em geral relacionadas à assistência técnica, adequação sanitária, gestão e logística. Por outro lado, esse cenário também permitiu o desenvolvimento de inovações locais para o atendimento das regras de acesso a esse mercado, como a adoção do "Vale Solidário", viabilizado pela cooperativa de crédito em um município de Minas Gerais para atender às dificuldades de capital de giro dos agricultores.

\subsection{OUTRAS CATEGORIAS}

As demais categorias identificadas neste estudo bibliométrico, somadas, contêm um total de treze artigos. A primeira delas é sobre estrutura escolar, que remete a análises sobre a estrutura física (instalações e equipamentos) e recursos humanos (cozinheiras, nutricionistas, membros do CAE e gestores da alimentação escolar) disponíveis para viabilizar a compra e, consequentemente, o preparo de alimentos adquiridos de agricultores familiares nas unidades escolares públicas (GONZÁLEZ-CHICA et al., 2013; FERNANDES; FONSECA; SILVA, 2014; FERREIRA et al., 2019). Os autores ressaltaram a importância de melhorias na estrutura física 
das escolas, na logística de recebimento/estocagem, na capacitação direcionada às cozinheiras e programas de educação alimentar nas escolas.

Outros três estudos foram categorizados como comparação internacional, por envolverem a problematização das experiências brasileiras a partir de iniciativas em curso em outros países na América Latina (BELIK; SOUZA, 2009; GRISA; CALDERÓN, 2019; SWENSSON, 2019). Destaca-se o fato do Brasil ser o único país da região que dispõe de um desenho de política alimentação escolar de caráter universal, com recursos integrais originários de orçamentos públicos próprios, em que todas as crianças da rede pública nos níveis da pré-escola e educação primária recebiam refeições. Nos demais países o que se observava é a adoção de programas focalizados, seja quanto à renda ou em termos geográficos, e dependentes de recursos da cooperação internacional.

$\mathrm{Na}$ categoria marco legal também foram identificados três artigos. Eles abordam sobre os processos políticos que levaram à reformulação do PNAE, com a promulgação da Lei no 11.947/2009 (PEIXINHO, 2013; SCHWARTZMAN et al., 2017; SILVA, 2019). As evidências indicaram que não houve um momento de mudança disruptiva, mas, sim, a ocorrência de eventos graduais, associados a fatores endógenos e exógenos, cujo acúmulo levou a transformações significativas em sua estrutura regulatória, na medida também em que o Estado foi se dotando das capacidades instrumentais necessárias para operacionalizar uma política bastante complexa. Entre os avanços apontados estão: expansão do público atendido, em razão da extensão do atendimento para toda a rede pública de educação básica e de jovens e adultos; elevação da disponibilidade orçamentária; fortalecimento dos CAEs; valorização do papel do profissional nutricionista; desconcentração dos fornecedores para o programa, propiciando maior potencial de geração de desenvolvimento local.

Dois textos foram enquadrados na categoria comunidades tradicionais, que aborda a relação do PNAE com públicos específicos do universo da agricultura familiar brasileira. Ambos tratam sobre comunidades quilombolas (SOUSA et al., 2013; CARVALHO; SILVA, 2014). Ambos enfatizaram a importância do PNAE no enfrentamento de situações de insegurança alimentar nessas comunidades, pois garante parte das necessidades alimentares e nutricionais dos indivíduos em idade escolar, ao mesmo tempo em que constitui fonte de renda e desenvolvimento local.

Por fim, dois textos realizaram uma pesquisa bibliográfica a respeito do estado da arte da pesquisa sobre o PNAE. Libermann e Bertolini (2015) usaram esse instrumento para verificar as principais tendências de pesquisa na área, que têm se caracterizado pelo estudo de estratégias de segurança alimentar e nutricional, envolvendo análise da composição e aceitação dos alimentos disponibilizados, bem como da produção e das condições de vida dos produtores rurais. Já Pedraza et al. (2018) verificaram na literatura diversos aspectos relacionados ao PNAE no território brasileiro, tais como: a inserção do nutricionista; a aquisição de alimentos da agricultura familiar; a implantação dos CAEs; e a execução dos cardápios.

\section{SÍNTESE DAS EVIDÊNCIAS ENCONTRADAS NA LITERATURA}

Com base na discussão até aqui realizada, foi possível compreender melhor as situações nas quais a relação entre Estado, alimentação escolar e agricultura familiar tem sido abordada nos veículos de produção científica no Brasil. Assim, obteve-se uma aproximação privilegiada sobre a realidade operacional do PNAE, uma vez que as análises demonstram aspectos concretos sobre os desafios e os potenciais associados à inserção desse público em mercados institucionais, ao mesmo tempo em que enfatizam fatores possíveis para a potencialização desse instrumento no 
território brasileiro. A partir dessa percepção, segue-se uma síntese geral das evidências encontradas:

i) a exigência de utilização dos valores repassados pelo FNDE para a compra mínima de $30 \%$ de produtos da agricultura familiar tem se constituído, na prática, mais como um ponto de chegada do que um ponto de partida. Os dados apontam uma trajetória perceptível de elevação do percentual médio em nível nacional, mas à medida que ele se eleva, surgem novas tensões e necessidades de complementação institucional, dadas as heterogeneidades políticas, sociais e geográficas que marcam o território brasileiro. Os estudos empíricos indicam uma quantidade considerável de fatores com potencial de impactar nos indicadores de desempenho dos municípios, que podem servir de orientação para o planejamento do programa em seus diversos níveis de execução, pois o simples uso do instrumento de chamadas públicas não garante a formalização dos contratos de compra junto aos agricultores.

ii) a abertura oficial de um novo canal de compras institucionais despertou uma série de dinâmicas organizacionais e formações de redes, incluindo entidades públicas e privadas, para potencializar a endogeneização dos ganhos possíveis propiciados pela legislação. Como o Brasil ainda possui uma quantidade grande de municípios pequenos, com parcelas significativas da população vivendo de atividades agrícolas, iniciativas como essa possuem um potencial não desprezível de dinamização dessas economias, com a injeção de novos recursos monetários via compra direta dos produtos. Para isso, as articulações no âmbito municipal e microrregional são fundamentais para a executabilidade das novas regras, estabelecendo padrões de comercialização mais próximos às realidades cotidianas da agricultura familiar.

iii) as profundas desigualdades territoriais em termos de infraestrutura para armazenamento e distribuição de alimentos naturais, potencial de resposta produtiva da agricultura familiar em distintas localidades e capacidades estatais de intervenção intersetorial nos níveis subnacionais da federação brasileira, evidenciam o desafio para a obtenção de resultados minimamente comuns de uma política dessa natureza em todo o território nacional. Isso torna necessário um acompanhamento constante dos indicadores para o aprimoramento dos instrumentos de coordenação federal.

iv) a nova legislação ampliou o grau de complexidade da gestão da política educacional no nível subnacional, ao introduzir um conjunto de novas regras para a execução do PNAE. Houve então a necessidade de aprendizados no plano organizacional para a assimilação dos novos procedimentos, além de reforçar a importância de pactuações do poder público com grupos da sociedade civil para garantir a efetivação do programa. Nesse contexto, o papel dos CAEs ganhou maior relevância, dada sua atuação tanto na mediação quanto no controle social.

v) a exigência de padrões mínimos de valor nutricional nas refeições escolares, com o aumento da restrição a certos tipos de alimentos processados, é algo que já vinha ocorrendo antes da Lei no 11.947/2009. Assim, a maior participação da agricultura familiar local no abastecimento de produtos para a alimentação dos estudantes favoreceu o atendimento dessas normativas, além de ressaltar ainda mais a importância do PNAE como política fundamental na garantia da segurança alimentar e nutricional e o DHAA em todos os municípios brasileiros.

vi) o novo desenho operacional do PNAE também fortalece a discussão da sustentabilidade e valorização ambiental. Além da relação mais harmoniosa com o meio ambiente que os sistemas de produção agrícola de base familiar necessitam, o programa passou a estimular a aquisição de alimentos orgânicos/agroecológicos, que exige o uso de insumos ambientalmente menos agressivos. Contudo, permanece a existência de limites aos grupos produtores para atender às exigências de quantidade, 
qualidade e preço, cuja superação passa pela disponibilidade de crédito, assistência técnica e certificação.

vii) a via da ação coletiva é constantemente destacada como elemento estratégico para que os agricultores familiares consigam atender às exigências legais e políticas no plano local. Nesse sentido, a organização desse segmento socioprodutivo em cooperativas e associações desponta como um caminho para superar a dispersão geográfica da produção, permitindo-Ihes maiores ganhos de escala, novas possibilidades de agroindustrialização e ampliação do poder de barganha nas negociações tanto com o poder público quanto com outros grupos privados.

viii) a introdução da obrigatoriedade de compra direta da agricultura familiar possibilitou ainda a inclusão de comunidades tradicionais como agentes fornecedores para o PNAE, sobretudo para escolas que atendem os próprios estudantes residentes dessas comunidades. Isso desponta então como um canal promissor de geração de renda para as famílias residentes, uma vez que grande parte delas trabalha com a atividade agrícola. Porém, esse tipo de inclusão exige uma série de cuidados e assessoramentos complementares para possibilitar que as organizações comunitárias consigam atender às exigências técnicas e burocráticas inerentes ao programa.

ix) outros agentes também têm demonstrado sua relevância no desempenho das unidades federativas nos indicadores do PNAE. Destaca-se o papel das nutricionistas, que elaboram os cardápios escolares que irão balizar os editais de compras públicas. As cozinheiras escolares também são relevantes, pois atuam diretamente no recebimento, preparo e distribuição das refeições, além de sua proximidade com os alunos consumidores dessas refeições. Nesse sentido, a percepção desses profissionais torna-se uma fonte importante de informações para o acompanhamento da efetividade do programa.

$x$ ) a reformulação do marco legal do PNAE derivou de um processo de discussões durante quase uma década, iniciada no âmbito do Consea, envolvendo atores dos mais distintos interesses. Portanto, foi uma mudança institucional dialogada, além de estar ancorada em outras alterações normativas que já vinham ocorrendo, e no caso da inclusão dos agricultores familiares, foram obedecidas as normativas já vigentes de outros programas voltados a esse grupo, como o PRONAF e o PAA.

\section{CONSIDERAÇÕES FINAIS}

Ao longo deste texto buscou-se observar o quanto a relação entre alimentação escolar e agricultura familiar tem despertado interesses de pesquisadores em distintas áreas do conhecimento científico. A análise bibliométrica demonstrou o crescimento desse interesse após a promulgação da Lei no 11.947/2009, que inseriu, entre outras coisas, a obrigatoriedade da destinação de um limite mínimo de 30\% dos repasses financeiros federais do PNAE para a aquisição de alimentos procedentes desse segmento socioprodutivo em todo o país. Esse novo marco regulatório sinaliza para um processo de mudança de paradigma no programa a partir de uma dupla perspectiva de intervenção estatal: melhorar a alimentação dos estudantes da rede pública de ensino, introduzindo alimentos mais saudáveis e condizentes com a realidade alimentar das comunidades escolares; e promover novas dinâmicas de desenvolvimento pela via do estímulo à expansão e diversificação produtiva da agricultura familiar nos territórios de incidência dessas ações.

A hipótese lançada de que as novas regras fariam com que o tema da agricultura familiar ganhasse maior importância relativa nas pesquisas sobre o PNAE foi validada com a constatação de que, enquanto até 2009 apenas 10,7\% dos artigos 
sobre alimentação escolar em periódicos brasileiros abordavam de alguma forma a temática da agricultura familiar. Após esse ano, com a promulgação da Lei n. 11.947, a relação aumentou para $33,3 \%$, mais que triplicando o interesse relativo entre as pesquisas sobre alimentação escolar no Brasil.

Além desta primeira constatação, foram levantadas as principais questões problematizadas no conjunto dos artigos selecionados. Para isso, optou-se pelo agrupamento dos textos em categorias temáticas, identificadas com base nas questões centrais de análise em cada pesquisa sobre a operacionalização do PNAE. Pode-se dizer a partir das análises evidenciadas que a conexão entre agricultura familiar e alimentação escolar abre um caminho importante para a promoção de hábitos alimentares saudáveis, com o oferecimento de cardápios de melhor qualidade nutricional e de acordo com a cultura produtiva regional no dia a dia de crianças e adolescentes de todo o país.

A necessidade de impulsionar a inclusão de alimentos produzidos regionalmente nos cardápios, viabilizando a entrada dos produtores locais nesse mercado, surge então como uma estratégia de diversificação e ampliação do valor nutricional da alimentação escolar, com vistas à melhoria nos processos educacionais. Contudo, embora o marco regulatório seja condição essencial, ele por si não é suficiente para viabilizar uma estratégia de intervenção governamental, sendo necessárias ações complementares para sua concreta efetivação, articuladas com outras estruturas setoriais. Ainda mais dada a heterogeneidade (geográfica, política, socioeconômica, cultural) que caracteriza o território brasileiro, tornando mais desafiadora a implementação de políticas nacionais com tamanha capilaridade como o PNAE. Por isso, ressalta-se aqui a importância de estudos interdisciplinares e avaliações permanentes que abordem esses processos e essas heterogeneidades no plano programático, visando melhorias sucessivas no alcance de seus objetivos junto à população brasileira.

\section{REFERÊNCIAS}

AMARAL, L. S. et al. Como a agricultura familiar se insere nos mercados agroalimentares? uma análise de experiências no RN. Revista Interface, v.15, 2018.

ANDRADE, J.; ARAÚJO, M. A.; SANTOS, P. Estudo sobre aquisição de alimentos da agricultura familiar para o PNAE. Revista Gestão e Sociedade, v.13, n.36, 2019.

ANTUNES, A. T.; VIVALDINI, M. Cooperativas e prefeituras do estado de São Paulo: uma análise da gestão colaborativa na cadeia de suprimento escolar. Revista Gestão e Planejamento, v.19, 2018.

ARAÚJO, A. L. et al. Contribution of the brazilian government purchasing programs PAA and PNAE - to strengthening family agriculture. Revista Espacios, v.38, 2017.

ARAUJO, L. R. S. et al. Alimentação escolar e agricultura familiar: análise de recursos empregados na compra de alimentos. Cadernos Saúde Pública, n.35, v.11, 2019.

BACCARIN, J. G. et al. Compras institucionais de produtos de agricultores familiares para alimentação escolar no estado de São Paulo sob a vigência da lei federal 11.947/2009. Revista Geografia, v.42, n.2, 2017.

BANDEIRA, L. M. et al. Análise dos pareceres conclusivos dos Conselhos de Alimentação Escolar sobre a execução do PNAE. Revista de Nutrição, v.26, 2013. 
BASSO, D.; LOPES, I. D.; AMARAL, V. R. Reflexões sobre a operacionalização do PNAE nas Escolas Estaduais de Passo Fundo-RS. Revista Redes, v.24, n.1, 2019.

BELIK, W.; CHAIM, N. A. O PNAE e a gestão municipal: eficiência administrativa, controle social e desenvolvimento local. Revista de Nutrição, v.22, n.5, 2009.

BELIK, W.; SOUZA, L. R. Algumas reflexões sobre os programas de alimentação escolar na América Latina. Revista Planejamento e Políticas Públicas, n. 33, 2009.

BEZERRA, O. M. et al. Promoção da aquisição de produtos da agricultura familiar para a alimentação escolar em Territórios da Cidadania de Minas Gerais e Espírito Santo. Revista de Nutrição, v.26, n.3, 2013.

CARVALHO, A. S.; SILVA, D. O. Perspectivas de segurança alimentar e nutricional no Quilombo de Tijuaçu, Brasil. Revista Interface, v.18, n.50, 2014.

CARVALHO, D. G. Licitações sustentáveis, alimentação escolar e desenvolvimento Regional: uma discussão sobre o poder de compra governamental a favor da sustentabilidade. Revista Planejamento e Políticas Públicas, n. 32, 2009.

CHAVES, L. G. et al. O programa de merenda escol a escolar de Campinas: análise do alcance e limitações do abastecimento regional. Revista de Nutrição, v.22, 2009.

CORREAA, R. S. et al. Family farming and school meals in Rio Grande do Sul. Revista Ciência Rural, v.49, n.2, 2019.

COSTA, B. L.; AMORIM Jr, P. C.; SILVA, M. G. As cooperativas de agricultura familiar e o mercado de compras governamentais em MG. Revista de Economia e Sociologia Rural, v.52, n.3, 2015.

CUNHA, W. A.; FREITAS, A. F.; SALGADO, R. Efeitos dos programas governamentais de aquisição de alimentos para a agricultura familiar em Espera Feliz, MG. Revista de Economia e Sociologia Rural, v.55, n.3, 2017.

D’ÁVILA, C.; SILVA, S. P. Segurança alimentar e desenvolvimento local: uma análise dos resultados do Programa de Aquisição de Alimentos (PAA) em Minas Gerais. Revista de Políticas Públicas, v. 15, n. 2, 2011.

ELIAS, L. P. et al. Impactos socioeconômicos do PNAE na agricultura familiar de Santa Catarina. Revista de Economia e Sociologia Rural, v.57, 2019.

ESTEVAM, D. O.; SALVARO, G. I.; SANTOS, V. J. Os desafios da inserção formal de produtos da agricultura familiar no mercado. Revista Redes, v.23, n.1, 2018.

FERIGOLLO, D. et al. Products purchased from family farming for school meals in the cities of Rio Grande do Sul. Revista Saúde Pública, v.51, 2017.

FERNANDES, D. M.; ENGEL, B. S. Agroindústrias familiares rurais: vantagens e desvantagens da legalização. Revista Redes, v.21, n.2, 2014. 
FERNANDES, A. G.; FONSECA, A. B.; SILVA, A. A. Alimentação escolar como espaço para educação em saúde: percepção das merendeiras do município do Rio de Janeiro. Revista Ciência e Saúde Coletiva, v.19, n.1, 2014.

FERREIRA, D. M. et al. Perception of the operating agents about the Brazilian National School Feeding Program. Cadernos de Saúde Pública, v.53, 2019.

FRANZONI, G. B.; SILVA, T. N. Inovação social e tecnologia social o caso da cadeia curta de agricultores familiares e a alimentação escolar em Porto Alegre/RS. Revista Desenvolvimento em Questão, n.37, 2016.

FREITAS, A. F.; FERREIRA, M. A..; FREITAS, A. F. A trajetória das organizações de agricultores familiares e a implementação de políticas públicas. Revista de Economia e Sociologia Rural, v.57, n.1, 2019.

GABRIEL, C. G. et al. Planejamento de cardápios para escolas públicas municipais. Revista de Nutrição, v.25, n.3, 2012.

GABRIEL, C. G.; GOULART, G.; CALVO, M. Gestão municipal do Programa Nacional de Alimentação Escolar nas capitais da região Sul. Revista de Nutrição, v.28, 2015.

GONÇALVES, H. V. et al. Family farming products on menus in school feeding. Revista Ciência Rural, v.45, n.12, 2015.

GONZÁLEZ-CHICA, D. A. et al. Percepção dos cozinheiros escolares sobre o processo de utilização de produtos orgânicos na alimentação escolar em municípios catarinenses. Revista de Nutrição, v.26, n.4, 2013.

GRISA, C.; CALDERÓN, P. E. Redes y alimentos: la participación de la agricultura familiar y campesina en la alimentación escolar del Brasil y Colombia. Revista Redes, v.24, n.1, 2019.

GRISA, C.; SCHNEIDER, S. Três gerações de políticas públicas para a agricultura familiar e formas de interação entre sociedade e estado no Brasil. Revista de Economia e Sociologia Rural, v. 52, 2014.

KROTH, D. C. et al. Análise da operacionalização da política de aquisição de alimentos da agricultura familiar por parte dos municípios. Revista Redes, v.24, 2019.

LIBERMANN, A. P.; BERTOLINI, G. R. Tendências de pesquisa em políticas públicas: uma avaliação do PNAE. Revista Ciência e Saúde Coletiva, v.20, n.11, 2015.

LIMA, E. E.; SOUSA, A. A. Alimentos orgânicos na produção de refeições escolares: limites e possibilidades em uma escola pública em Florianópolis. Revista de Nutrição, v.24, n.2, 2011.

LOPES, B. J.; DOULA, S. M. Políticas públicas e governança: análise do Programa Nacional de Alimentação Escolar no Sudeste. Revista Espacios, v.37, 2016.

LOPES, B. J.; DOULA, S. M. Deficiências no processo de compras governamentais do PNAE e seus impactos no desenvolvimento local. Revista Desenvolvimento em Questão, n.45, 2019. 
MACHADO, P. M. et al. Compra de alimentos da agricultura familiar pelo PNAE. Revista Ciência e Saúde Coletiva, v.23, n.12, 2018.

MALUF, R. et al. Nutrition-sensitive agriculture and the promotion of food and nutrition sovereignty and security in Brazil. Revista Ciência e Saúde Coletiva, v.20, n.8, 2015.

MARQUES, A. A. et al. Reflexões de agricultores familiares sobre a dinâmica de fornecimento de seus produtos para a alimentação escolar. Revista Saúde e Sociedade, v.23, n.4, 2014.

MARTINS, L. F.; LIMA, S. M. Programa de Aquisição da Produção da Agricultura no Papa/DF: inovação gerencial e tecnológica em sistemas familiares, 2009-2016. Revista de Economia e Sociologia Rural, v.55, n.3, 2017.

MELO, M. N.; SÁ, R. M.; MELO FILHO, D. A. Sustentabilidade de um programa de alimentação escolar bem-sucedido. Revista Ciência e Saúde Coletiva, v.21, 2016.

MONEGO, E. T. et al. Produção e potencial agrícolas de alimentos destinados à alimentação escolar em Goiás e no Distrito Federal. Revista de Nutrição, v.26, 2013.

MOSSMANN, M. P. et al. Interface between family farming and school seeding: barriers and coping mechanisms from the perspective of different social actors in Southern Brazil. Revista de Economia e Sociologia Rural, v.55, n.2, 2017.

NASCIMENTO, S. G. et al. Produção agroecológica e segurança alimentar e nutricional (Brasil). Revista de Ciências Agrárias, v.42, n.1, 2019.

OLIVEIRA, L. G.; BATALHA, M. O.; PETTAN, K. B. Comparative assessment of the food purchase program and the national school feeding program's impact in Ubá, Minas Gerais. Revista Ciência Rural, v.47, n.1, 2015.

PACÍFICO, D. A. Os sistemas locais de ação pública e a sua origem nas redes sociotécnicas: a territorialização do Plano Brasil Sem Miséria rural. Revista Extensão Rural, v. 24, n. 3, 2017.

PAIVA, J. B.; FREITAS, M. C.; SANTOS, L. A. Hábitos alimentares regionais no Programa Nacional de Alimentação Escolar. Revista de Nutrição, v.25, n.2, 2012.

PEDRAZA, D. F. et al. Avaliação do Programa Nacional de Alimentação Escolar: revisão da literatura. Revista Ciência e Saúde Coletiva, v.23, n.5, 2018.

PEIXINHO, A. M. A trajetória do PNAE no período de 2003-2010: relato do gestor nacional. Revista Ciência e Saúde Coletiva, v.18, n.4, 2013.

REAL, L. C. V.; SCHNEIDER, S. O uso de programas públicos de alimentação na reaproximação do pequeno produtor com o consumidor: o caso do programa de alimentação escolar. Revista Estudo e Debate, v.18, n.2, 2011.

RIBEIRO, A. L.; CERATTI, S.; BROCH, D. T. Programa Nacional de Alimentação Escolar (PNAE) e a participação da agricultura familiar em municípios do RS. Revista Gestão e Desenvolvimento em Contexto, v.1, n.1, 2013. 
SA, S. S. et al. A interface entre alimentação escolar e agricultura familiar em Jampruca e Mathias Lobato, Minas Gerais. Revista Espacios, v.38, n.46, 2017.

SAMBUICHI, R. H. R. et al. Programa de Aquisição de Alimentos e Segurança Alimentar. Brasília: Ipea, 2019. (Texto para Discussão, n. 2482).

SANTOS, F. et al. Avaliação da inserção de alimentos orgânicos provenientes da agricultura familiar na alimentação escolar, em municípios dos territórios rurais do RS. Revista Ciência e Saúde Coletiva, v.19, n.5, 2014.

SARAIVA, E. B. et al. Panorama da compra de alimentos da agricultura familiar para o PNAE. Revista Ciência e Saúde Coletiva, v.18, 2013.

SCHABARUM, J. C.; TRICHES, R. M. Aquisição de produtos da agricultura familiar em municípios paranaenses: análise dos produtos comercializados e dos preços praticados. Revista de Economia e Sociologia Rural, v.57, n.1, 2019.

SCHWARTZMAN, F.; MORA, CI. A.; BOGUS, C. M.; VILLAR, B. S. Antecedentes e elementos da vinculação do programa de alimentação escolar do Brasil com a agricultura familiar. Cadernos de Saúde Pública, v.33, n.12, 2017.

SILVA, A. P.; SOUSA, A. A. Alimentos orgânicos da agricultura familiar no PNAE de Santa Catarina. Revista de Nutrição, v.26, n.6, 2013.

SILVA, S. P. Mediação social e incidência territorial de políticas públicas de desenvolvimento rural no Médio Jequitinhonha/MG. Cadernos Gestão Pública e Cidadania, n. 65, 2014a.

SILVA, S. P. A trajetória histórica da segurança alimentar e nutricional na agenda política nacional. Brasília: Ipea, 2014b. (TD no 1953).

SILVA, S. P. A dinâmica das políticas públicas de desenvolvimento rural e sua incidência territorial: uma análise do Pronaf no território Vale do Mucuri/MG. Extensão Rural, Santa Maria, v. 22, n. 2, p.60-78, abr./jun. 2015.

SILVA, S. P. Agricultura familiar e território: aspectos conceituais e analíticos sobre a multifuncionalidade e a pluriatividade. Revista Campo Território, v. 11, 2016.

SILVA, S. P. O campo de pesquisa da economia solidária no Brasil: abordagens metodológicas e dimensões analíticas. Brasília: Ipea, 2018. (TD oㅡ 2.361)

SILVA, S. P. Trajetória e padrões de mudança institucional no Programa Nacional de Alimentação Escolar. Brasília: Ipea, 2019. (TD no 2.529)

SILVA, S. P.; VALADARES, A. A. Segurança alimentar e nutricional. In: IPEA. Desafios da nação. Brasília: Ipea, 2018. v. 2.

SILVA, M. G.; DIAS, M. M.; AMORIM Jr, P. C. Mudanças organizacionais em empreendimentos de agricultura familiar a partir do acesso ao Programa Nacional de Alimentação Escolar. Revista de Economia e Sociologia Rural, v.52, n.3, 2015. 
SIQUEIRA, R. L. et al. Análise da incorporação da perspectiva do direito humano à alimentação adequada no desenho institucional do programa nacional de alimentação escolar. Revista Ciência e Saúde Coletiva, v.19, n.1, 2014.

SILVERIO, G. A.; SOUSA, A. A. Organic foods from family farms in the National School Food Program. Revista de Nutrição, v.27, n.3, 2014.

SOARES, P. et al. Potencialidades e dificuldades para o abastecimento da alimentação escolar mediante a aquisição de alimentos da agricultura familiar em um município brasileiro. Revista Ciência e Saúde Coletiva, v.20, n.6, 2015.

SOARES, P. et al. Programa Nacional de Alimentação Escolar como promotor de Sistemas Alimentares Locais. Revista Ciência e Saúde Coletiva, v.23, 2018.

SOUSA, L. M. et al. Alimentação escolar nas comunidades quilombolas: desafios e potencialidades. Revista Ciência e Saúde Coletiva, v.18, n.4, 2013.

SOUSA, A. A.; SILVA, A. P.; AZEVEDO, E.; RAMOS, M. O. Cardápios e sustentabilidade: ensaio sobre o PNAE. Revista de Nutrição, v.28, n.2, 2015.

SOUZA, V. M.; VILLAR, B. S. Acquisition of family farm foods in municipalities of São Paulo state. Revista de Nutrição, v.32, 2019.

SOUZA-ESQUERDO, V. F.; BERGAMASCO, S. M. Análise sobre o acesso aos programas de políticas públicas da agricultura familiar nos municípios do circuito das frutas (SP). Revista de Economia e Sociologia Rural, v.52, n.1, 2014.

SWENSSON, L. F. Institutional food procurement programmes and producer organizations. Revista Redes, v.24, n.1, 2019.

TEO, C. R.; MONTEIRO, C. A. Marco legal do Programa Nacional de Alimentação Escolar. Revista de Nutrição, v.25, n.5, 2012.

TRICHES, R. M.; SCHNEIDER, S. Alimentação escolar e agricultura familiar: reconectando o consumo à produção. Revista Saúde e Sociedade, v.19, n.4, 2010.

TRICHES, R. M.; KILIAN, L. Papel dos atores sociais na aquisição de produtos da agricultura familiar para alimentação escolar em municípios paranaenses. Revista Redes, v.21, n.3, 2016.

TRICHES, R. M.; SILVESTRI, F. Adequação das chamadas públicas para aquisição de produtos da agricultura familiar para a alimentação escolar. Revista Desenvolvimento em Questão, n.44, 2018.

TRICHES, R. M. et al. Condicionantes e limitantes na aquisição de produtos da agricultura familiar pelo PNAE no Paraná. Revista Redes, v.24, n.1, 2019.

TRIVELLATO, P. T. et al. Food and nutrition (in)security in families of farmers who supply the National School Feeding Program. Revista de Nutrição, v.32, 2019. 
TURPIN, M. E. A alimentação escolar como fator de desenvolvimento local por meio do apoio aos agricultores familiares. Revista Segurança Alimentar e Nutricional, v.16, n.2, 2009.

VALADARES, A. A. et al. Agricultura familiar e abastecimento alimentar no contexto do Covid-19. Brasília: Ipea, 2020. (Nota Técnica, no 69)

VIANNA, R. P.; TERESO, M. J. O programa de merenda escolar de Campinas: análise do alcance e limitações do abastecimento regional. Revista de Nutrição, v.13, 2000.

VIEIRA, F. S. A produção em saúde do Ipea: contribuição à promoção do desenvolvimento socioeconômico brasileiro. Brasília: Ipea, 2019. (Texto para Discussão, no 2.496)

VILELA, K. et al. Implementation of the national school nourishment program in the Brazilian Federal institution of Education of Bahia. Revista Ciência Rural, v.49, 2019.

VILLAR, B. S. et al. Situação dos municípios de São Paulo com relação à compra direta de produtos da agricultura familiar para o PNAE. Revista Brasileira Epidemiologia, v.16, n.1, 2013.

VILHENA, M. R.; ALVES Jr, A.; FREITAS, A. F. Produtos da sociobiodiversidade no PAA e PNAE. Revista de Extensão e Estudos Rurais, v.8, n.1, 2019.

WAGNER, D.; GEHLEN, I.; SCHULT, G. Agricultura Familiar, políticas públicas e cidadania. Revista Redes, v.21, n.3, 2016. 